\title{
The Opportunity of Linked Data for the European Higher Education Area
}

\author{
Guillermo Vega-Gorgojo, Thanassis Tiropanis, and David E. Millard
}

\begin{abstract}
The european higher education area (EHEA) aims to create a more comparable, compatible and coherent space of Higher Education in Europe. After a decade of reforms, most of the required instruments are already in place, so the next years are key to complete the implementation and consolidation of the EHEA. However, data challenges pose a threat to pivotal EHEA processes such as credit recognition that require institutions to cooperate and integrate their datasets scattered across diverse repositories in multiple data formats. Linked Data principles and practices have the potential to address such difficulties, as demonstrated with the emergence of a global data space -the Web of Data. This paper first discusses the benefits of Linked Data for the emergent EHEA, devising a new set of Linked Data-driven scenarios that leverage current EHEA processes. Further, a set of preliminary Linked Data deployments is examined to assess the advantages and identify the challenges ahead in the EHEA landscape.
\end{abstract}

Index Terms-European higher education area (EHEA), linked data, semantic web, technology-enhanced learning (TEL).

\section{INTRODUCTION}

The so-called Bologna Process aims to create the European Higher Education Area (EHEA), involving 47 countries across Europe. As stated in the Bologna Declaration [1] the goals of the emergent EHEA are to facilitate the compatibility and comparability of study programmes, to encourage the mobility of students and staff, increase employability and promote the attractiveness of the EHEA world-wide. After more than ten years from the beginning of the Bologna Process, national legislation and regulation reforms of Higher Education have been completed in most countries [2]. As a result, most of the fundamental elements of the EHEA are now in place including the adoption of a three-cycle degree structure, a credit-based system, standards and guidelines for quality assurance, and the definition of qualification frameworks based on learning outcomes, as summarized in Fig. 1.

However, the achievement of certain strategic goals as

Manuscript received June 25, 2014; revised September 12, 2014. This research has been partially funded by the European Commission BYTE project FP7 GA 619551, the Spanish Ministry of Economy and Competitiveness project TIN2011-28308-C03-02, and the Autonomous Government of Castilla and León, Spain, project VA301B11-2.

Guillermo Vega-Gorgojo is with the Department of Informatics, University of Oslo, Norway (e-mail: guiveg@ifi.uio.no).

Thanassis Tiropanis is with the School of Electronics and Computer Science, University of Southampton, UK (e-mail: tt2@ecs.soton.ac.uk).

David E. Millard is with the School of Electronics and Computer Science, University of Southampton, UK (e-mail: dem@ecs.soton.ac.uk). envisioned in the EHEA has appeared to be especially challenging [3]. Specifically, mobility within the EHEA has not increased substantially, study programmes should be more compatible and comparable, and the employability goal remains elusive [4]. One of the reasons is that many EHEA processes such as credit recognition or quality assurance are very demanding due to excess of bureaucracy and human intervention [5], [6]. Furthermore, these processes typically require the cooperation of multiple EHEA institutions, thus posing challenges on the integration and the interoperability of data dispersed in institutional repositories [7].

Similar difficulties for integrating datasets from different sources have been reported many times in other domains such as enterprise systems integration [8]. Particularly noteworthy is the Linking Open Data project that has led to the creation of a global data space based on an increasing number of contributors [9], [10]. The goal of this project was to bootstrap the development of a Web of Data based on common standards and rules such as the Linked Data principles [11] for publishing data on the Web. The success of this initiative has been such that even governments have begun to release some of their datasets as Linked Data with the aim of increased transparency and efficient administrative procedures [12].

In the Higher Education field some preliminary works propose the use of Linked Data technologies to address the challenges for Technology-Enhanced Learning (TEL), as well as supporting activities such as student admission [7], [13], [14]. In the wider context of the EHEA the impact of such technologies should be even more significant given the requirements for cross-institutional exchange and integration of data. Despite this, there are no studies in the literature motivating the use of Linked Data technologies in the EHEA to the extent of the authors' knowledge. This paper aims to discuss the potential of Linked Data for supporting EHEA processes. In this regard, the use of these technologies for addressing the data requirements of the emergent EHEA is analysed and we propose a set of Linked Data-driven scenarios. Furthermore, a set of preliminary Linked Data deployments is revised to assess the benefits of these technologies, as well as to identify the challenges ahead to better support Europe's Higher Education landscape.

\section{THE OPPORTUNITY OF LINKED DATA FOR THE EHEA}

This section begins with a discussion of the suitability of Linked Data to support EHEA processes. Then, a set of Linked Data-driven scenarios is outlined, thus illustrating the potential of these technologies for the realization of the EHEA. 


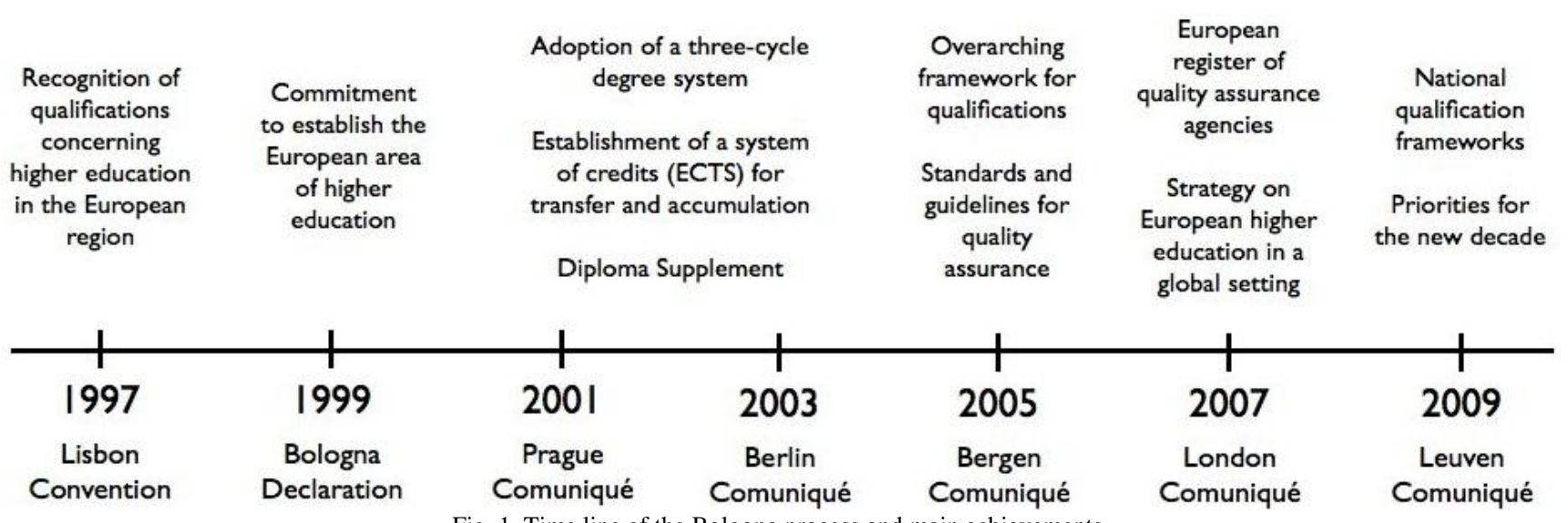

\section{A. The Potential of Linked Data for the Implementation of the EHEA}

Linked Data technologies provide for the exposure of data as well as links (relationships) among data on the Web [10], [15]. Exposed data may originate from databases, Web pages or files such as excel spreadsheets in different organisations. Linked Data technologies make use of Uniform Resource Identifiers (URIs) to identify data and relationships and they provide for describing, storing and querying Linked Data based on standards such as RDF [16], RDFa [17] and

SPARQL [18]. There are proposals and methodologies on how relational database tables can be exposed as Linked Data [19] and proposed rules for the emerging Web of Data [11]. These rules encourage the reuse of URIs where possible in order to make it easy to obtain the same type of data from different sources and to perform aggregation and simple reasoning. In addition, there are requirements on supporting the 'dereferencing' of URIs, i.e. obtaining further information about the corresponding data or relationship [11].
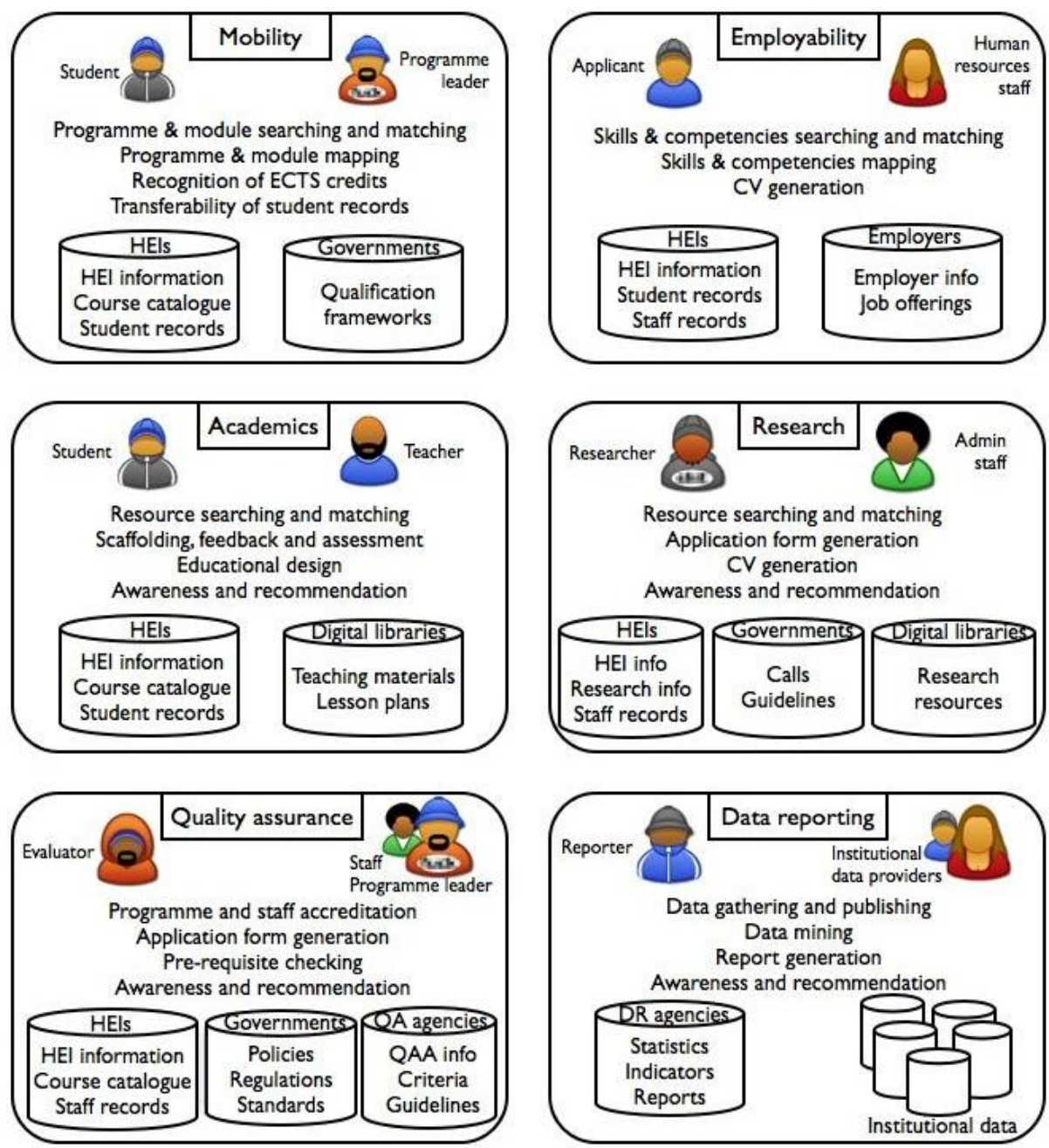

Fig. 2. Potential linked data-driven scenarios fro the EHEA.

Linked Data technologies can support data interoperability and integration and thus provide for building more advanced applications over the Web. In addition, a Linked Data infrastructure can pave the way for adding more expressive 
descriptions of exposed data and could, in turn, support advanced inferencing and more intelligent applications. In this way, the Linked Data represent a bottom-up approach to implementing the Semantic Web vision [20]; in this approach the value of data exposure, interoperability and integration is significant enough to be given priority over building consensus on common vocabularies and ontologies.

It has been reported that a Linked Data infrastructure across Higher Education Institutions (HEIs) can help the sector meet certain challenges [7]. In particular, the findings of a survey that was conducted by the UK JISC-funded SemTech project in 2008-2009 suggest that such infrastructures could address challenges related to learning, teaching and supporting HEI processes [13]. According to [7] relevant learning and teaching challenges that could benefit from Linked Data infrastructures include 1) course creation, delivery and revision, 2) access to teaching and learning material across institutions, and 3) cross-curricular activities in emerging areas; challenges related to Higher Education processes involve 1) curriculum development or alignment, 2) degree programme accreditation, and 3) cross-institutional collaboration.

These Higher Education challenges, which Linked Data infrastructures could help to address, are also related to the international cooperation envisioned by the Bologna Process and to the programme and curriculum reform for convergence of the EHEA. Specifically, data exposure in interoperable formats as defined in the Semantic Web can support advanced data processing and integration applications. In addition, the emergence of vocabularies and, where necessary, vocabulary mappings for the EHEA could further increase the potential for data integration. The Linked Data design recommendations [11] show that vocabulary convergence could improve interoperability significantly, although it is not a prerequisite for Linked Data applications. Existing tools identified by the SemTech project [13] could provide for efficient exposure and mapping of Linked Data.

\section{A. Linked Data-Driven EHEA Scenarios}

In order to exploit the potential of Linked Data for the further development of the EHEA, stakeholders should expose their key datasets into the Linked Data cloud. For instance, HEIs could offer as Linked Data their course catalogues [21] that include information on programmes, qualifications, modules, learning outcomes, ECTS credits and so on. Likewise, data reporting agencies could expose their statistics, indicators and reports as Linked Data. Table I summarizes the key datasets that could be offered by the main stakeholders in the EHEA.

Note that some institutions are beginning to expose some of their datasets as Linked Data. For instance, the University of Southampton has recently launched the Open Data Service (http://data.southampton.ac.uk/) and other European universities are also embracing Linked Data -Linked Universities (http://linkeduniversities.org) lists some of these institutions. Moreover, Eurostat now offers a Linked Data version (http://eurostat.linked-statistics.org/) of their datasets and some governments and administrations have begun to release data from the public sector following the Linked Data principles. These pioneering initiatives should be followed by other institutions, thus contributing to constitute an incipient EHEA Web of Data.

TABLE I: MAIN STAKEHOLDERS IN THE EHEA AND KEY DATASETS

\begin{tabular}{|c|c|}
\hline Stakeholder & Key datasets \\
\hline Governments & $\begin{array}{l}\text { - Regulations, guidelines } \\
\text { - Project, grant, position calls } \\
\text { - Indicators, statistics, reports }\end{array}$ \\
\hline Higher Education Institutions & $\begin{array}{l}\text { - Institutional information } \\
\text { - Course catalogue } \\
\text { - Student records } \\
\text { - Staff records }\end{array}$ \\
\hline Quality assurance agencies & $\begin{array}{l}\text { - Agency information } \\
\text { - Criteria } \\
\text { - Indicators, statistics, reports }\end{array}$ \\
\hline Employers & $\begin{array}{l}\text { - Employer information } \\
\text { - Employment offerings }\end{array}$ \\
\hline Data reporting agencies & - Indicators, statistics, reports \\
\hline Digital libraries and repositories & $\begin{array}{l}\text { - Research resources } \\
\text { - Teaching materials }\end{array}$ \\
\hline
\end{tabular}

With the gradual exposure of institutional datasets, it is envisioned that there will be an emergence of a new breed of Linked Data-driven applications in the EHEA landscape. As a result, some current human-intensive tasks could be automated or at least better supported with technology. Considering the main application areas of the EHEA, a new set of scenarios can be devised, as summarized in Fig. 2:

- Mobility. The availability of HEI course catalogues enables the development of expert systems for searching programmes and modules. This way, students' mobility could be fostered allowing them to find appropriate degrees for their interests. Moreover, the exposure of other datasets, e.g. student records, can facilitate additional features such as checking programme prerequisites or suggesting mobility grants.

From the perspective of a HEI, students' mobility involves many bureaucratic processes like programme admission, recognition of ECTS credits or the transferability of students' records that could be supported with Linked Data-based tools. For instance, programme mappings via qualification frameworks and learning outcomes can help to assess the similarity of modules across institutions, thus facilitating recognition processes.

- Employability. In this area there is ample room for improvement, since qualifications are not always comprehensible for employers and they are commonly unaware of instruments such as the Diploma Supplement [4]. A promising solution is the description of qualifications in terms of learning outcomes, thus enabling the development of job matching services based on skills and competencies. With an EHEA Web of Data, employers can even obtain evidence of the acquisition of a particular competency. Further, generation of curricula could be partially automated gathering data from student or staff records and HEI course catalogues.

- Academics. The potential of Linked Data for Higher Education was supported in [13], identifying various suitable TEL scenarios. One example is the search and matching of teaching resources across institutions. Student tutoring and scaffolding can also be leveraged by recommending materials for an assignment or finding a tutor to support students' activities. In addition, supporting scenarios for programme and module design 
can be devised: identifying niches for new modules, comparing programmes across institutions or integrating information scattered across different datasets for documentation purposes.

- Research. In this case, many scenarios can be found requiring some kind of resource searching and matching such as researchers, groups, papers, projects, conferences, grant calls and so on. Moreover, notification and recommendation services are especially suitable for awareness and to deal with deadlines. Further, highly bureaucratized processes like project proposal preparations could be eased with semi-automatic generation of application forms and curricula.

- Quality assurance. Programme and staff accreditation activities are becoming ubiquitous in the emergent EHEA. These processes tend to be data-intensive, requiring applicants to prepare exhaustive applications and evaluators to revise the documentation and find evidence of claims. Thus, a Linked Data approach could help to gather data dispersed in different sources, automatically generate some forms such as curricula, perform a prerequisite checking of an application and so on.

- Data reporting. This area is critical to assess the state of development of the EHEA [3] and Linked Data seems a very promising approach to enhance both availability and quality of the data. Adoption of established Linked Data publishing practices by all stakeholders in the EHEA can enormously facilitate the work of data reporting agencies. For instance, data gathering should be eased and usage of common formats may enhance data comparability, thus leveraging the generation of statistics, indicators and reports. Further, exposure of institutional datasets seems a promising field for data mining processes.

\section{EMERGING LINKED DATA DEVELOPMENTS IN THE EHEA}

After discussing the opportunity of Linked Data for the realization of the EHEA, this section describes a set of ongoing projects that illustrate the benefits of Linked Data technologies in some of the key areas of the EHEA. The first three projects describe university-wide infrastructures that utilise Linked Data, while the second three projects present novel TEL applications that exploit Linked Data technologies.

\section{A. Open Linked Data from the Open University}

Similar to other universities in Europe, the Open University (OU) exposes some of their institutional datasets as Linked Data [22]. Specifically, research publications, teaching material, course descriptions and people profiles are currently available. Whenever possible, popular and well-established vocabularies are employed, e.g. the Bibliographic Ontology (BIBO http://bibliontology.com/specification) and Friend of a Friend (FOAF http://xmlns.com/foaf/spec/) - as well as educational domain vocabularies such as AIISO (http://vocab.org/aiiso/) for the organization of academic institutions. All these data are available both through a SPARQL endpoint and as RDF.

The technical infrastructure consists on a set of components that extract RDF from existing OU repositories, load this RDF into a triple store and make it available through the Web. Beyond the exposure of datasets, some applications that use these data have been developed, such as graph generators, a building locator, and a course recommendation system. Overall, these preliminary applications require little effort to exploit OU datasets and to link to external sources such as DBpedia (http://dbpedia.org/), thus demonstrating the benefits of the Linked Data approach.

\section{B. The Southampton Learning Environment}

The Southampton Learning Environment (SLE) project is a TEL initiative across the University of Southampton as part of its Curriculum Innovation Programme. It involves a large group of stakeholders from different faculties, the University's information and communication technologies (ICT) professional services department and the students' union. SLE uses Linked Data technologies for the integration of data in repositories across or outside the University with the aim of improving the student and staff experience, of making graduates more employable and of enabling remote, blended and distance learning alleviating the teaching space problem [23].

SLE is in the process of deploying an RDF layer over repositories such as timetable information, virtual learning environments, eAssessment, library and computer assisted assessment. The integration of external and cloud-based services and repositories such as GoogleDocs, DropBox, TurnitIn and Delicious is also envisaged. The deployment of SPARQL endpoints to support this RDF layer involves a separation between repositories providing authoritative and non-authoritative university data and a separation between private and public university data sources. SLE also considers community engagement models for the development of applications over this layer; as a first iteration an Open Linked Data portal (http://data.southampton.ac.uk) was implemented to this end, providing an one stop access point to data sources and an application store to host applications that were crowdsourced from across or outside the University. The portal features a number of innovative applications include a university amenities map and a number of smartphone-based applications.

\section{Talis Aspire}

Talis Aspire [24] is a resource management system widely adopted by HEIs in the UK. With this system, teachers can prescribe learning materials to students through a conventional Web interface; behind the scenes, RDF triples are produced and persisted to a centralized triple store, enabling the linking of resources across institutions. Whilst $\mathrm{BIBO}$ is used to annotate scholar materials, academic and course information is described with AIISO. With such a volume of structured data, Talis Aspire shines at discovering resources via browsing, searching and recommendation mechanisms.

\section{MEducator}

The mEducator project (http://www.meducator.net/) aims to create a federated repository of health educational materials. Existing medical education repositories are not 
interoperable due to competing data schemas and heterogenous API interfaces. Ref. [25] proposes a general architecture for integrating existing Web educational resources (data and services) and exposing them as Linked Data: each repository is annotated semantically, then resource annotations are retrieved and stored in a triple store, and finally automatically enriched with dataset interlinking. This approach is followed in mEducator, exposing as Linked Data the resulting dataset describing resources according to the mEducator Resource RDF schema (http://purl.org/meducator/ns/). mEducator exemplifies an evolutionary development that takes profit of Linked Data to provide a federated repository without requiring the substitution of its parts that remain operable.

\section{E. Quiz Games with Greek DBpedia}

Ref. [26] presents a web game that uses datasets derived from the Greek DBpedia. It provides several quiz types and is purposed for educational purposes by native speakers, taking profit from the DBpedia internationalization effort. The application architecture is relatively simple, generating thousands of questions from the Greek DBpedia using 30 seed SPARQL queries to retrieve facts belonging to geography, history, and so on. This game has been tested in a primary education pilot study, obtaining a positive reception. This project illustrates the serendipitous reusability enabled by the Web of Data: the creation of the DBpedia is a collaborative effort that can be easily exploited in novel and unanticipated ways by taking profit of its underlying data structure.

\section{F. SEEK-AT-WD}

SEEK-AT-WD is a Linked Data-based search system of tools for Higher Education [27]. Given the number of tools that can be employed to leverage current learning settings, this system aims to provide guidance to teachers. It is a follow-up of Ontoolsearch [28], a system that supports semantic search of educational tools that can be visually constructed using the terms defined in the vocabulary Ontoolcole [29]. Since Ontoolsearch employs its own isolated dataset, it is difficult and costly to keep tool information up to date. In contrast, SEEK-AT-WD exploits the Web of Data to gather tool descriptions available as Linked Data in DBpedia, Freebase (http://www.freebase.com/) and other open datasets. Ontoolcole is still employed to provide a homogenous view of educational tools, developing a set of mappings to translatedescriptions in external data sources to thisvocabulary. As a result, this proposal is much more sustainable, taking profit of available information in the Web of Data to keep updated the tool dataset in an automated way.

\section{DISCUSSION}

The scenarios and the specific projects that are outlined in the previous sections illustrate that Linked Data technologies are not only appealing, but they can serve to leverage key activities of the EHEA. However, many key datasets for the development of the EHEA remain hidden in institutional realms, thus forming part of the so-called Deep Web [30]. In other cases, datasets are publicly available on the Web, typically in HTML format; however, they lack the required structure and semantics for more automated processing (e.g. searching mechanisms) and are largely disconnected from other data sources. In consequence, independent reports on the state of the EHEA [4] as well as the strategic agenda [3] emphasize the need of better data exposure both for spreading the EHEA and for monitoring achievements.

Anyway, the situation is changing and many ongoing efforts are devoted to exposing existing datasets as Linked Data. In this regard, demonstrators such as the Open Linked Data are of great importance to induce other EHEA institutions to follow this path. While early experiences report that setting up a Linked Data service is not trivial [31], this situation is likely to improve in the near future since tools are rapidly maturing and more clear guidelines and best practices are crystallizing [32]. For instance, EHEA institutions can choose among different types of data publishers depending on the need of supporting legacy data sources or not: one option is the use of a triple store that natively supports RDF; so-called RDFizers can convert legacy formats into RDF; mapping tools such as D2R Server [33] can be employed to expose relational databases as Linked Data; in addition, some commercial data storage platforms like Virtuoso (http://virtuoso.openlinksw.com/) offer a Linked Data interface. The mEducator project illustrates the creation of a federated dataset from legacy repositories though the use of Linked Data.

Data modelling is another key element for publishing datasets, and vocabularies provide the necessary structure to enable data processing by applications. Vocabularies should be open and shared by a community of users in order to facilitate data integration processes. In the outlined projects, bibliographic resources are described with BIBO, people profiles with FOAF and so on. Noteworthy, learning specific vocabularies such as AIISO, mEducator schema and Ontoolcole are also employed. Currently, there is a rise of ongoing proposals for describing specific domains in the EHEA such as competencies, outcomes, learning opportunities and assessment -see for example the ICOPER Reference

Model (http://www.icoper.org/results/reference-model) and the ASPECT Vocabulary Bank for Education (http://aspect.vocman.com/vbe/). Other remarkable initiatives include the Bowlogna ontology [34] for academic settings, the Learning Resource Metadata Initiative (LRMI http://www.lrmi.net/) for learning objects and XCRI (http://www.xcri.co.uk/) for describing courses, although it is increasingly difficult to keep track of all vocabulary offerings. Indeed, some of these proposals compete and may overlap thus requiring schema mappings and data fusion techniques to provide for data interoperability. In the mid-term vocabulary convergence can be expected in many cases. In this regard, the role of standardization bodies such as the CEN Workshop on Learning Technologies (CEN provides specifications, agreements, guidelines, and recommendations of special relevance to the EHEA, see http://www.cen.eu/CEN/sectors/sectors/isss/activity/Pages/ wslt.aspx) is of great importance to establish a common ground. 
As a note of caution, Linked Data technologies are yet to address challenges related to scalability. Federated queries over a large number of Linked Data repositories across institutions is yet to be addressed although there has been significant work in the area of federated databases and early work on supporting distributed queries in the Web of Data [35]-[37]. Until these technologies are mature enough to support federated queries on a large scale, triple stores are expected to store data from different sources and therefore reduce the requirement for distributed queries and repository federation. In addition, the privacy of certain information in Linked Data repositories (e.g. student or staff records) is another cause of concern. Although access control can be imposed on repositories, more elaborate control that will ensure that private information cannot be queried or inferred (privacy of RDF graphs) is currently supported only by specific environments [38].

Once datasets are openly available, new applications can be developed to consume these data to provide relevant functionalities for the EHEA. In the simplest case, applications can be designed to explore Linked Data sources, allowing users to browse, search or visualize a dataset, e.g. the charting application of the Open Linked Data. However, the real value of Linked Data comes out when interlinking datasets and exploiting the Web of Data. In this regard, platforms like Talis Aspire effectively create a web of scholar resources, allowing the discovery and recommendation of materials by exploiting the links (resources by topic, course, popularity, etc.). Moreover, the SLE project shows how Linked Data technologies can profoundly impact TEL by opening up institutional data, allowing more transparent and flexible use of data within an institution, and creating opportunities for creative third-party applications. Additionally, the Greek DBpedia quiz game and the SEEK-AT-WD projects illustrate how the Web of Data can be used as a data source, reducing the overall authoring effort and improving the sustainability of the solution. While the outlined cases are mainly driven by a single organization, cross-institutional developments are expected in the near future, especially in mobility, employability and quality assurance EHEA scenarios that require further coordination actions. One early example is the Linked Universities project (http://linkeduniversities.org/) that aims to create a common space of universities' Linked Data thus reducing the overall effort.

As a wrap-up of this discussion, Table II extracts the main challenges that can serve as a roadmap for better supporting the emergent EHEA with Linked Data technologies.

TABLE II: LINKED DATA CHALLENGES AHEAD FOR SUPPORTING THE EMERGENT EHEA

\begin{tabular}{|c|c|}
\hline Challenge & Comment \\
\hline Exposure of more datasets & $\begin{array}{c}\text { Steady progress and better supporting } \\
\text { tools }\end{array}$ \\
\hline $\begin{array}{c}\text { Availability of vocabularies } \\
\text { Linked Data technology } \\
\text { concerns }\end{array}$ & $\begin{array}{c}\text { New proposals and vocabulary } \\
\text { convergence }\end{array}$ \\
\hline Appealing applications & Demonstrators already available \\
\hline $\begin{array}{c}\text { Cross-institutional } \\
\text { developments }\end{array}$ & $\begin{array}{c}\text { More value for the EHEA, inception } \\
\text { phase }\end{array}$ \\
\hline
\end{tabular}

\section{CONCLusions}

The Bologna Process towards the construction of the EHEA has been demonstrated to be especially challenging. Steady progress over the past decade has led to a number of milestones that include programme and curriculum reforms as well as instruments such as the ECTS, the Diploma Supplement and qualification frameworks. Despite this, pivotal EHEA processes, e.g. credit recognition or student mobility, are specially demanding due to the need for cooperation among institutions and for overcoming the difficulties of the integration and interoperation of data scattered across diverse repositories.

Emergent Linked Data technologies have the potential to address the main data challenges of the EHEA. In this sense, initial deployments in the EHEA demonstrate the viability of the Linked Data approach and its benefits for accessing, integrating and consuming data in an homogeneous way. As some pioneering EHEA institutions are releasing some of their repositories, an incipient EHEA Web of Data is growing. Preliminary applications show that retrieval of data is consistent across all providers, benefitting from the interconnection and structuring of Linked Data sources. Furthermore, some of these developments such as SLE or Talis Aspire are especially relevant to the TEL domain, particularly to show the integration of disparate repositories through a Linked Data layer.

In the near future, further progress is expected in the key areas of the EHEA, driven by Linked Data technologies. For instance, fostering students' mobility requires assessing the characteristics and prerequisites of programme degrees, the recognition of ECTS credits and competencies among HEIs, and facilitating the transfer of student records. Exposing the key datasets as Linked Data enables the creation of expert information systems and advanced data processing applications that automate, or at least better support, the aforementioned tasks. If the challenges highlighted above are adequately addressed, Linked Data technologies are expected to enable a significant step towards the construction of the EHEA.

\section{REFERENCES}

[1] EHEA Ministers, "The Bologna declaration of 19 June 1999," Ministers responsible for Higher Education in the EHEA, Bologna, Italy, Joint declaration, Jun. 1999.

[2] P. Zgaga, "1999-2010 - The making of Bologna," 1999-2010 Achievements, Challenges and Perspectives, Ministry of Science and Research, Austria, and Ministry of Education and Culture, Hungary, 2010, pp. 6-9.

[3] EHEA Ministers, "The Bologna process 2020 - The European higher education area in the new decade," Ministers responsible for Higher Education in the EHEA, Leuven/Louvain-la-Neuve, Belgium, Comuniqué, Apr. 2009.

[4] CHEPS, INCHER-Kassel and ECOTEC consortium, "The bologna process independent assessment. The first decade of working on the European higher education area," Assessment report, 2010.

[5] A. Veiga and A. Amaral, "How does the Bologna process challenge the national traditions of higher education institutions?" Cultural Perspectives on Higher Education, Springer, 2008, pp. 245-263.

[6] ESU, "Towards 2020: A student-centred bologna process," European Students' Union (ESU), Report, Dec. 2008.

[7] T. Tiropanis, H. Davis, D. Millard, and M. Weal, "Semantic technologies for learning and teaching in the Web 2.0 era," IEEE Intelligent Systems, vol. 24, no. 6, pp. 49-53, 2009.

[8] J. M. Myerson, Enterprise Systems Integration, 2nd ed., Boca Raton, FL, USA: Auerbach Publications, 2002. 
[9] C. Bizer, "The emerging web of linked data," IEEE Intelligent Systems, vol. 24, no. 5, pp. 87-92, 2009.

[10] C. Bizer, T. Heath, and T. Berners-Lee, "Linked data - the story so far," International Journal on Semantic Web and Information Systems, vol. 5, no. 3, pp. 1-22, 2009.

[11] T. Berners-Lee, "Linked data - Design issues," 2006.

[12] N. Shadbolt, "Open government data: Making it real," in Proc. the Open Government Data Camp 2011, Warsaw, Poland, Oct. 2011.

[13] T. Tiropanis, H. Davis, D. Millard, M. Weal, S. White, and G. Wills, "Semantic technologies in learning and teaching (SemTech)," University of Southampton, JISC report, 2009.

[14] P. Miller, "Linked data horizon scan," Joint Information Systems Committee (JISC), JISC report, Jan. 2010.

[15] T. Heath and C. Bizer, Linked Data: Evolving the Web into a Global DataSpace, Morgan \& Claypool, 2011.

[16] G. Klyne and J. Carroll, "Resource Description framework (RDF): concepts and abstract syntax," W3C, Recommendation, Feb. 2004.

[17] B. Adida, I. Herman, M. Sporny, and M. Birbeck, "RDFa 1.1 Primer Rich Structured Data Markup for Web Documents," W3C, W3C Working Group Note, Jun. 2012.

[18] E. Prud'hommeaux and A. Seaborne, "SPARQL query language for RDF," W3C, Recommendation, Jan. 2008.

[19] D. Allemang and J. A. Hendler, Semantic Web for the Working Ontologist: Effective Modeling in RDFS and OWL, Burlington, MA, USA: Morgan Kaufmann, 2008.

[20] T. Berners-Lee, J. Hendler, and O. Lassila, "The semantic web," Scientific American, vol. 284, no. 5, pp. 34-43, 2001.

[21] European Commission, ECTS Users' Guide, Brussels, Belgium: Luxembourg: Office for Official Publications of the European Communities, Feb. 2009.

[22] F. Zablith, M. Fernandez, and M. Rowe, "The OU linked open data: Production and consumption," in Proc. the 1st International Workshop on eLearning Approaches for Linked Data Age (Linked Learning 2011), 8th Extended Semantic Web Conference (ESWC2011), Heraklion, Greece, May 2011, pp. 35-49.

[23] S. White and H. C. Davis, "Making it rich and personal: crafting an institutional personal learning environment," International Journal of Virtual and Personal Learning Environments, vol. 2, no. 4, pp. 23-39, 2012.

[24] C. Clarke, "A resource list management tool for undergraduate students based on linked open data principles," presented at the 6th European Semantic Web Conference (ESWC2009), Heraklion, Greece, 2009.

[25] S. Dietze, H. Yu, D. Giordano, E. Kaldoudi, N. Dovrolis, and D. Taibi, "Linked education: interlinking educational resources and the web of data," in Proc. the 27th Annual ACM Symposium on Applied Computing (SAC '12), 2012, pp. 366-371.

[26] C. Bratsas, D. Chrysou, E. Eftychiadou, D. Kontokostas, P. Bamidis, and I. Antoniou, "Semantic web game based learning: An I18n approach with Greek DBpedia," presented at the 2nd International Workshop on Learning and Education with the Web of Data (LiLe-2012 at WWW- 2012), Lyon, France, Apr. 2012.

[27] A. Ruiz-Calleja, G. Vega-Gorgojo, J. Asensio-Pérez, M. Bote-Lorenzo, E. Gómez-Sánchez, and C. Alario-Hoyos, "A linked data approach for the discovery of educational ICT tools in the web of data," Computers \& Education, vol. 59, no. 3, pp. 952-962, 2012.

[28] G. Vega-Gorgojo, M. L. Bote-Lorenzo, J. I. Asensio-Pérez, E. Gómez-Sánchez, Y. A. Dimitriadis, and I. M. Jorrín-Abellán, "Semantic search of tools for collaborative learning with the Ontoolsearch system," Computers \& Education, vol. 54, no. 4, pp. 835-848, 2010.

[29] Y. A. Dimitriadis et al., "Ontoolcole: Supporting educators in the semantic search of CSCL tools," Journal of Universal Computer Science (JUCS), vol. 14, no. 1, pp. 27-58, 2008.

[30] B. He, M. Patel, Z. Zhang, and K. C.-C. Chang, "Accessing the deep web," Communications of the ACM, vol. 50, no. 5, pp. 95-101, 2007.

[31] J. Hannemann and J. Kett, "Linked data for libraries," presented at the 76th IFLA World Library and Information Congress, Gothenburg, Sweden, Aug. 2010.

[32] L. Dodds and I. Davis, Linked Data Patterns: A Pattern Catalogue for Modelling, Publishing, and Consuming Linked Data, 2012.
[33] C. Bizer and R. Cyganiak, "D2R Server - Publishing relational databases on the Semantic Web," presented at the 5th International Semantic Web Conference (ISWC 2006), Athens, GA, USA, Nov. 2006.

[34] G. Demartini, J. G. I. Enchev, and P. Cudré-Mauroux, “The Bowlogna ontology: Fostering open curricula and agile knowledge bases for Europe's Higher Education landscape," Semantic Web, vol. 4, no. 1, pp. $53-63,2013$.

[35] S. Schenk and J. Petrak, "Sesame RDF repository extensions for remote querying," presented at the 7th Znalosti Conference, Bratislava, Slovakia, Jan. 2008

[36] X. Wang, T. Tiropanis, and H. Davis, "Evaluating graph traversal algorithms for distributed sparql query optimization," presented at the Joint International Semantic Technology Conference, Hangzhou, China, Dec. 2011.

[37] A. Schwarte, P. Haase, K. Hose, R. Schenkel, and M. Schmidt, "Fedx: Optimization techniques for federated query processing on linked data," presented at the 10th International Semantic Web Conference (ISWC 2011), Bonn, Germany, Oct. 2011.

[38] C. Farkas, V. Gowadia, A. Jain, and D. Roy, "From xml to rdf: Syntax, semantics, security, and integrity (invited paper)," Security Management, Integrity, and Internal Control in Information Systems, pp. $41-55,2006$

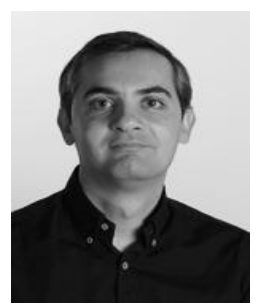

Guillermo Vega-Gorgojo is a postdoctoral researcher in the Logic and Intelligent Data Group at the University of Oslo in Norway. His research interests include linked data, semantic web, big data, search interfaces, distributed systems and e-learning. Guillermo has a $\mathrm{PhD}$ in telecommunications engineering from the University of Valladolid in Spain. He is also a member of the Intelligent \& Cooperative Systems Research Group at the University of Valladolid, researching on disruptive technologies in Higher Education.

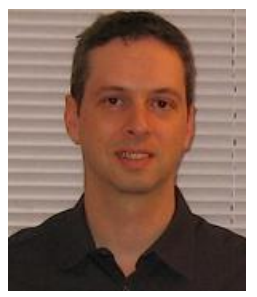

Thanassis Tiropanis is a senior lecturer in the Web and Internet Science Group at the University of Southampton in the UK. His research interests include web science, social networks, distributed linked data infrastructures and linked data for higher education. Thanassis has worked for many years in research on Web infrastructures, network and service management, business-to-business applications, and collaboration infrastructures for formal and informal learning. He holds a diping in computer engineering and informatics from the University of Patras, Greece, and a $\mathrm{PhD}$ in computer science from University College London. He is a senior member of the IEEE, a chartered IT professional with BCS, a fellow of the Higher Education Academy in the UK, a professional member of ACM and a member of the Technical Chamber of Greece.

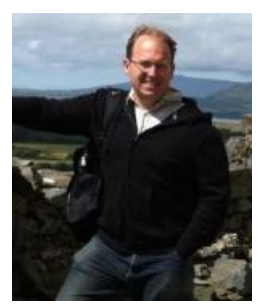

David E. Millard is a senior lecturer in the Web and Internet Science Group at the University of Southampton in the UK. His research interests have long involved hypertext and Web research - first the area of open, adaptive, and contextual hypermedia, and more recently Web 2.0 , the semantic web, knowledge and narrative interfaces, and the impact of Web literacy on e-learning and mobile learning. $\mathrm{He}$ is interested in the ways that people use information systems in the wild, and how we can use emergent social, organizational, and semantic structures to help them make sense of their world. David has a $\mathrm{PhD}$ in computer science from the University of Southampton. 